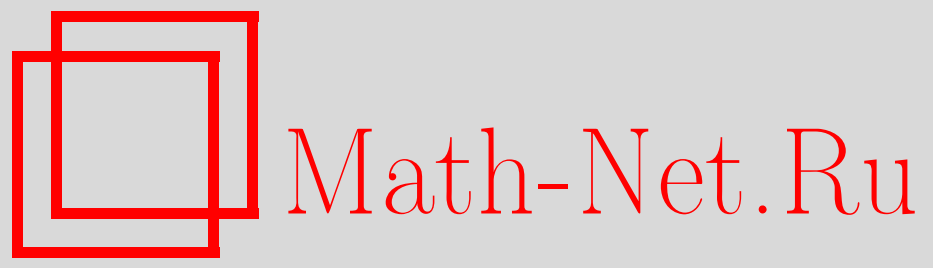

Обцероссийский математический портал

М. З. Гараев, Об одном ряде с простыми нулями $\zeta(s), M a-$ тем. заметки, 2003, том 73, выпуск 4, 627-629

DOI: https://doi.org/10.4213/mzm625

Использование Общероссийского математического портала Math-Net.Ru подразумевает, что вы прочитали и согласны с пользовательским соглашением http://www.mathnet.ru/rus/agreement

Параметры загрузки:

IP : 3.80 .253 .173

26 апреля 2023 г., $17: 21: 52$ 


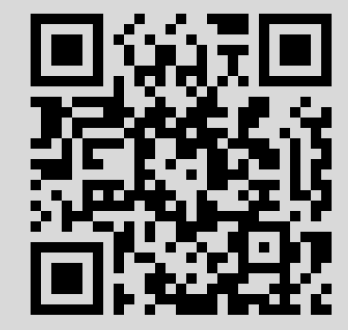




\section{ОБ ОДНОМ РЯДЕ С ПРОСТЫМИ НУЛЯМИ $\zeta(s)$}

\section{М.3. Гараев}

1. Введение. Пусть $N(T)$ обозначает число нулей $\zeta(s)$ внутри полосы $0<\operatorname{Im} s<T$, а $N_{1}(T)$ обозначает число простых нулей $\zeta(s)$ вида $s=1 / 2+i t, 0<t<T$. Через $\rho=\beta+i \gamma$ мы обозначим нули $\zeta(s)$. Далее в тексте константа в знаке Виноградова 》 абсолютная.

На с. 374 книги Титчмарша [1] под редакцией и с дополнениями Д. Р. Хиз-Брауна доказано, что если гипотеза Римана верна и если все нули $\zeta(s)$ простые, то ряд

$$
\sum\left|\rho \zeta^{\prime}(\rho)\right|^{-1}
$$

расходится. В настоящей работе мы улучшаем этот результат, доказьвая при этом следующую безусловную теорему.

Теорема. Имеет место оченка

$$
\sum_{0<\operatorname{Im} \rho<T}^{*}\left|\rho \zeta^{\prime}(\rho)\right|^{-1} \gg(\log T)^{1 / 2},
$$

əде $\sum^{*}$ означает, что суммирование ведется только по простым нулям $\zeta(s)$.

Из теоремы, в частности, следует, что ряд $\sum^{*}\left|\rho \zeta^{\prime}(\rho)\right|^{-1}$ расходится.

Для доказательства теоремы будем пользоваться оценкой

$$
N_{1}(T) \geqslant 0.342 N(T),
$$

которая справедлива для всех достаточно болшшх $T$ (см. [2]). Нам также потребуется следующая лемма.

ЛЕмма. Пусть $S(t)$ - комплекснозначная дважсди непрерывно дифференцируемая функчия на отрезке $\left[t_{0}, t_{k}\right]$. Пусть, далее, $t_{0}<t_{1}<\cdots<t_{k} u$

Тогда

$$
S\left(t_{j}\right)=0, \quad 0 \leqslant j \leqslant k .
$$

$$
\sum_{1 \leqslant j \leqslant k}\left|S^{\prime}\left(t_{j}\right)\right| \leqslant \sqrt{2} \int_{t_{0}}^{t_{k}}\left|S^{\prime \prime}(t)\right| d t .
$$

ДОКАЗАТЕЛЬСТво. Пусть $S(t)=A(t)+i B(t)$, где $A(t)$ и $B(t)$ - действительнозначные функции. Тогда при $0 \leqslant j \leqslant k$ имеем $A\left(t_{j}\right)=B\left(t_{j}\right)=0$. Поэтому для каждого $j, 0 \leqslant j<k$, существует $\tau_{j} \in\left(t_{j}, t_{j+1}\right)$ такое, что $A^{\prime}\left(\tau_{j}\right)=0$. Следовательно,

$$
A^{\prime}\left(t_{j+1}\right)=\int_{\tau_{j}}^{t_{j+1}} A^{\prime \prime}(t) d t
$$


откуда

$$
\left|A^{\prime}\left(t_{j+1}\right)\right| \leqslant \int_{\tau_{j}}^{t_{j+1}}\left|A^{\prime \prime}(t)\right| d t \leqslant \int_{t_{j}}^{t_{j+1}}\left|A^{\prime \prime}(t)\right| d t .
$$

Суммируя обе части по $j$ в пределах $0 \leqslant j \leqslant k-1$, получим

$$
\sum_{1 \leqslant j \leqslant k}\left|A^{\prime}\left(t_{j}\right)\right| \leqslant \int_{t_{0}}^{t_{k}}\left|A^{\prime \prime}(t)\right| d t
$$

Аналогично,

$$
\sum_{1 \leqslant j \leqslant k}\left|B^{\prime}\left(t_{j}\right)\right| \leqslant \int_{t_{0}}^{t_{k}}\left|B^{\prime \prime}(t)\right| d t
$$

Поэтому

$$
\begin{aligned}
\sum_{1 \leqslant j \leqslant k}\left|S^{\prime}\left(t_{j}\right)\right| & \leqslant \sum_{1 \leqslant j \leqslant k}\left|A^{\prime}\left(t_{j}\right)\right|+\sum_{1 \leqslant j \leqslant k}\left|B^{\prime}\left(t_{j}\right)\right| \leqslant \int_{t_{0}}^{t_{k}}\left(\left|A^{\prime \prime}(t)\right|+\left|B^{\prime \prime}(t)\right|\right) d t \\
& \leqslant \sqrt{2} \int_{t_{0}}^{t_{k}}\left|A^{\prime \prime}(t)+i B^{\prime \prime}(t)\right| d t=\sqrt{2} \int_{t_{0}}^{t_{k}}\left|S^{\prime \prime}(t)\right| d t .
\end{aligned}
$$

2. Доказательство теоремы. Пусть $n$ - достаточно большое натуральное число. Пусть $\sum_{\gamma}^{* *}$ означает, что суммирование ведется только по тем $\gamma$, для которых $1 / 2+i \gamma$ является простым нулем $\zeta(s)$. Ясно, что для доказательства теоремы достаточно установить оценку

$$
\sum_{10^{n}<\gamma<10^{n+1}}^{* *}\left|\gamma \zeta^{\prime}\left(\frac{1}{2}+i \gamma\right)\right|^{-1} \gg n^{-1 / 2}
$$

Для доказательства же последней оценки применим к сумме

$$
\sum_{10^{n}<\gamma<10^{n+1}}^{* *}\left|\zeta^{\prime}\left(\frac{1}{2}+i \gamma\right)\right|
$$

лемму, полагая в ней $S(t)=\zeta(1 / 2+i t)$. Тогда получим

$$
\sum_{10^{n}<\gamma<10^{n+1}}^{* *}\left|\zeta^{\prime}\left(\frac{1}{2}+i \gamma\right)\right| \ll \int_{1}^{10^{n+1}}\left|\zeta^{\prime \prime}\left(\frac{1}{2}+i t\right)\right| d t .
$$

Применение неравенства Гёльдера дает

$$
\int_{1}^{10^{n+1}}\left|\zeta^{\prime \prime}\left(\frac{1}{2}+i t\right)\right| d t \ll\left(10^{n} \int_{1}^{10^{n+1}}\left|\zeta^{\prime \prime}\left(\frac{1}{2}+i t\right)\right|^{2} d t\right)^{1 / 2}
$$

Из приближенного функционального уравнения для $\zeta^{\prime \prime}(1 / 2+i t)$ (см. [3, с. 90-92]) путем стандартных вычислений легко вывести оценку

$$
\int_{1}^{10^{n+1}}\left|\zeta^{\prime \prime}\left(\frac{1}{2}+i t\right)\right|^{2} d t \ll n^{5} 10^{n}
$$

Поэтому из (2) и (3) следует, что

$$
\sum_{10^{n}<\gamma<10^{n+1}}^{* *}\left|\zeta^{\prime}\left(\frac{1}{2}+i t\right)\right| \ll n^{5 / 2} 10^{n} .
$$


Согласно (1) число слагаемых в левой части неравенства (4) имеет порядок $n 10^{n}$. Следовательно,

$$
n 10^{n} \ll \sum_{10^{n}<\gamma<10^{n+1}}^{* *}\left|\zeta^{\prime}\left(\frac{1}{2}+i \gamma\right)\right|^{-1 / 2}\left|\zeta^{\prime}\left(\frac{1}{2}+i \gamma\right)\right|^{1 / 2} .
$$

Применяя неравенство Гёльдера и используя (4), получим

$$
n 10^{n} \ll\left(\sum_{10^{n}<\gamma<10^{n+1}}^{* *}\left|\zeta^{\prime}\left(\frac{1}{2}+i \gamma\right)\right|^{-1}\right)^{1 / 2}\left(n^{5 / 2} 10^{n}\right)^{1 / 2}
$$

откуда

$$
\sum_{10^{n}<\gamma<10^{n+1}}^{* *}\left|\zeta^{\prime}\left(\frac{1}{2}+i \gamma\right)\right|^{-1} \gg 10^{n} n^{-1 / 2} .
$$

Поэтому

$$
\sum_{10^{n}<\gamma<10^{n+1}}^{* *}\left|\gamma \zeta^{\prime}\left(\frac{1}{2}+i \gamma\right)\right|^{-1} \gg n^{-1 / 2}
$$

Теорема доказана.

\section{СПИСОК ЦИТИРОВАННОЙ ЛИТЕРАТУРЫ}

1. Titchmarsh E.C. The Theory of the Riemann Zeta-Function. Oxford: Clarendon Press, 1986. 2. Heath-Brown D. R. // Bull. London Math. Soc. 1979. V. 11. P. 17-18. 3. Воронин C. М., Карацуба А. А. Дзета-функция Римана. М.: Физматлит, 1994.

Институт математики и механики АН Азербайджана, г. Баку 